UPR-1253-T

\title{
F-Theory Compactifications with Multiple U(1)-Factors: Addendum
}

\author{
Mirjam Cvetič ${ }^{1,2}$, Denis Klevers ${ }^{1}$ and Hernan Piragua ${ }^{1}$ \\ ${ }^{1}$ Department of Physics and Astronomy, \\ University of Pennsylvania, Philadelphia, PA 19104-6396, USA \\ ${ }^{2}$ Center for Applied Mathematics and Theoretical Physics, \\ University of Maribor, Maribor, Slovenia \\ cvetic at cvetic.hep.upenn.edu, klevers at sas.upenn.edu, hpiragua at sas.upenn.edu
}

\begin{abstract}
The purpose of this note is to extend the results obtained in [1] in two ways. First, the six-dimensional F-theory compactifications with $\mathrm{U}(1) \times \mathrm{U}(1)$ gauge symmetry on elliptic Calabi-Yau threefolds, constructed as a hypersurface in $d P_{2}$ fibered over the base $B=\mathbb{P}^{2}$ [1] , are generalized to Calabi-Yau threefolds elliptically fibered over an arbitrary twodimensional base $B$. While the representations of the matter hypermultiplets remain unchanged, their multiplicities are calculated for an arbitrary $B$. Second, for a specific non-generic subset of such Calabi-Yau threefolds we engineer $\mathrm{SU}(5) \times \mathrm{U}(1) \times \mathrm{U}(1)$ gauge symmetry. We summarize the hypermultiplet matter representations, which remain the same as for the choice of the base $B=\mathbb{P}^{2}$ [2], and determine their multiplicities for an arbitrary $B$. We also verify that the obtained spectra cancel anomalies both for $\mathrm{U}(1) \times \mathrm{U}(1)$ and $\mathrm{SU}(5) \times \mathrm{U}(1) \times \mathrm{U}(1)$.
\end{abstract}

July, 2013 


\section{Contents}

1 Introduction

2 Compactifications for a General Base: $\mathrm{U}(1) \times \mathrm{U}(1) \quad 2$

2.1 Construction of the Fibration . . . . . . . . . . . . . . . . . . 2

2.2 Hypermultiplet Matter Representations and Multiplicities . . . . . . . 4

2.3 Anomaly Cancellation ................... 5

3 Compactifications for a General Base: $\mathrm{SU}(5) \times \mathrm{U}(1)^{2} 6$

3.1 Hypermultiplet Matter Representations . . . . . . . . . . . . . . . . 8

3.2 Hypermultiplet Matter Multiplicities . . . . . . . . . . . . . . . . . . 9 9

3.3 Anomaly cancellation . . . . . . . . . . . . . . 11

\section{Introduction}

In this Addendum we extend the results of [1] to constructions of elliptically fibered Calabi Yau threefolds $\hat{X}$ with Mordell-Weil group of rank two for an arbitrary twodimensional base $B$, leading to a most general construction of six-dimensional F-theory compactifications with $\mathrm{U}(1) \times \mathrm{U}(1)$ gauge symmetry. While the explicit examples in [1] were given for the base $B=\mathbb{P}^{2}$, a generalization to an arbitrary base turns out to depend only on the information about the three divisors in the base: the anti-canonical divisor of $B$ and two more divisors $\mathcal{S}_{9}$ and $\mathcal{S}_{7}$ that are projections with respect to $\pi: \hat{X} \rightarrow B$ to the homology of the base $B$ of particular intersections of the divisor classes $S_{Q}, S_{R}$ and $S_{P}$ of the rational sections $\hat{s}_{Q}, \hat{s}_{R}$ and the zero section $\hat{s}_{P}$, namely $\mathcal{S}_{9}=\pi\left(S_{P} \cdot S_{R}\right)$ and $\mathcal{S}_{7}=\pi\left(S_{Q} \cdot S_{R}\right)$

The hypermultiplet matter representations appear at codimension two singularities where the fiber degenerates in a way that is independent of the base $B$. Therefore their representations under $\mathrm{U}(1) \times \mathrm{U}(1)$ gauge symmetry, which were determined in [1], remain the same for an arbitrary base $B$. On the other hand, their multiplicities do depend on the base choice, and in this Addendum we extend the calculation of hypermultiplet matter multiplicities to an arbitrary base and verify anomaly cancellation. These results therefore determine hypermultiplet matter representations and their multiplicities for a general six-dimensional compactification of F-theory with $\mathrm{U}(1) \times \mathrm{U}(1)$ gauge symmetry.

Furthermore, in the original paper we gave a specific toric example of a non-generic elliptic Calabi Yau threefold with the gauge group $\mathrm{SU}(5) \times \mathrm{U}(1) \times \mathrm{U}(1)$. The purpose was to show that it was possible to add a non-Abelian group on divisors in the base, along with the Abelian sector $\mathrm{U}(1) \times \mathrm{U}(1)$. The hypermultiplet matter representations, which again depend only on the codimension two singularity structure of the fiber, were 
subsequently given in [2]. Here we engineer $\mathrm{SU}(5) \times \mathrm{U}(1) \times \mathrm{U}(1)$ gauge symmetry on a non-generic elliptically fibered Calabi-Yau threefold over an arbitrary base $B$. We derive the multiplicities of the hypermultiplet matter representations and verify anomaly cancellation. In this analysis only the information on one additional vertical divisor $\mathcal{S}_{\mathrm{SU}(5)}$, which is associated with the $\mathrm{SU}(5)$ gauge symmetry, is needed.

We follow closely the notation of the subsequent paper [2] where four-dimensional F-theory compactifications with $\mathrm{U}(1) \times \mathrm{U}(1)$ and $\mathrm{SU}(5) \times \mathrm{U}(1) \times \mathrm{U}(1)$ gauge symmetry were studied. We have also taken some formulae, tables and figures from this paper.

For related work on F-theory compactifications on Calabi-Yau manifolds with rank two Mordell-Weil group, see [3, 4, 5, 6].

The Addendum is structured in the following way. In Section 2 we systematically construct Calabi-Yau threefolds with $\mathrm{U}(1) \times \mathrm{U}(1)$ gauge symmetry, which are elliptically fibered over an arbitrary base, calculate the multiplicities of all hypermultiplet matter representations and verify anomaly cancellation. In Section 3 we summarize the spectrum for a specific non-generic fibration with $\mathrm{SU}(5) \times \mathrm{U}(1) \times \mathrm{U}(1)$ gauge symmetry, and calculate multiplicities for Calabi-Yau threefolds with an arbitrary base. We also show that these spectra cancel six-dimensional anomalies.

\section{Compactifications for a General Base: $\mathrm{U}(1) \times \mathrm{U}(1)$}

In this Section we work out the spectrum of a general F-theory compactification to six dimensions on elliptically fibered Calabi-Yau threefolds $\pi: \hat{X} \rightarrow B$ realizing a rank two Abelian sector. Consistency of the theory is shown at the level of anomaly cancellation.

\subsection{Construction of the Fibration}

The general elliptic fiber with a rank two Mordell-Weil group is given by the generic elliptic curve $\mathcal{E}$ in $d P_{2}$ [1, 3], which takes the following form:

$p=u\left(s_{1} u^{2} e_{1}^{2} e_{2}^{2}+s_{2} u v e_{1} e_{2}^{2}+s_{3} v^{2} e_{2}^{2}+s_{5} u w e_{1}^{2} e_{2}+s_{6} v w e_{1} e_{2}+s_{8} w^{2} e_{1}^{2}\right)+s_{7} v^{2} w e_{2}+s_{9} v w^{2} e_{1}$,

where $\left[u: v: w: e_{1}: e_{2}\right]$ are the homogeneous coordinates on $d P_{2}$ and the $s_{i}$ 's are numbers in $\mathbb{C}$.

The elliptic fibrations of this curve over the base $B=\mathbb{P}^{2}$ have been constructed in [1. In this Section, we extend the construction to an arbitrary base $B$. Here we closely follow the derivation and the notation of [2]. These elliptic threefolds $\hat{X}$ can be described as a hypersurface in a four-dimensional ambient space.

We begin by constructing this ambient space. It is given by a $d P_{2}$-fibration over the 
two-dimensional base $B$,

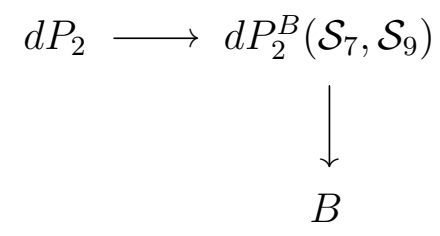

In this fibration the homogeneous coordinates of $d P_{2}$ are lifted to sections of line bundles over the base $B$. Using the three $\mathbb{C}^{*}$-actions on $d P_{2}$ three of these coordinates can be chosen to transform in the trivial line bundle on the base without loss of generality. We can choose the following assignments of line bundles for the remaining two homogeneous coordinates:

$$
u \in \mathcal{O}_{B}\left(\mathcal{S}_{9}+\left[K_{B}\right]\right), \quad v \in \mathcal{O}_{B}\left(\mathcal{S}_{9}-\mathcal{S}_{7}\right),
$$

where $\left[K_{B}\right]$ is the canonical bundle on $B$ and $\mathcal{S}_{7}, \mathcal{S}_{9}$ are arbitrary divisors on $B$. We note that $(2.2)$ is the natural generalization of eq. (3.30) in [1].

Next, we construct an elliptically fibered Calabi-Yau threefold with its general elliptic fiber in $d P_{2}$. It is described as the hypersurface (2.1) in the ambient space (2.2). The Calabi-Yau condition enforces that the coefficients $s_{i}$ are lifted to sections of appropriate line bundles on $B$. To see this, we calculate the total Chern class of the ambient space via adjunction,

$c\left(d P_{2}^{B}\right)=c(B)\left(1+H-E_{1}-E_{2}+\mathcal{S}_{9}-\left[K_{B}^{-1}\right]\right)\left(1+H-E_{2}+\mathcal{S}_{9}-\mathcal{S}_{7}\right)\left(1+H-E_{1}\right)\left(1+E_{1}\right)\left(1+E_{2}\right)$,

where $H, E_{1}$ and $E_{2}$ are the three divisor classes on $d P_{2}$, c.f., eq. (3.24) in [1] as well as eq. (2.2) of [2]. This yields the following anti-canonical bundle:

$$
K_{d P_{2}^{B}}^{-1}=\mathcal{O}\left(3 H-E_{1}-E_{2}+2 \mathcal{S}_{9}-\mathcal{S}_{7}\right)
$$

where for brevity of notation we suppressed the dependence on $\mathcal{S}_{7}, \mathcal{S}_{9}$ on the left side of (2.4) and (2.5). Requiring that the hypersurface (2.1) is a section of the anti-canonical bundle, we obtain the following assignment of line bundles for the coefficients $s_{i}$ :

\begin{tabular}{c|ccc|c} 
Section & Bundle & & Section & Bundle \\
\cline { 2 - 2 }$u$ & $\mathcal{O}\left(H-E_{1}-E_{2}+\mathcal{S}_{9}+\left[K_{B}\right]\right)$ & & $s_{1}$ & $\mathcal{O}\left(3\left[K_{B}^{-1}\right]-\mathcal{S}_{7}-\mathcal{S}_{9}\right)$ \\
$v$ & $\mathcal{O}\left(H-E_{2}+\mathcal{S}_{9}-\mathcal{S}_{7}\right)$ & & $s_{2}$ & $\mathcal{O}\left(2\left[K_{B}^{-1}\right]-\mathcal{S}_{9}\right)$ \\
$w$ & $\mathcal{O}\left(H-E_{1}\right)$ & $s_{3}$ & $\mathcal{O}\left(\left[K_{B}^{-1}\right]+\mathcal{S}_{7}-\mathcal{S}_{9}\right)$ \\
$e_{1}$ & $\mathcal{O}\left(E_{1}\right)$ & $s_{5}$ & $\mathcal{O}\left(2\left[K_{B}^{-1}\right]-\mathcal{S}_{7}\right)$ \\
$e_{2}$ & $\mathcal{O}\left(E_{2}\right)$ & $s_{6}$ & $\mathcal{O}\left(\left[K_{B}^{-1}\right]\right)$ \\
& & $s_{7}$ & $\mathcal{O}\left(\mathcal{S}_{7}\right)$ \\
& & $s_{8}$ & $\mathcal{O}\left(\left[K_{B}^{-1}\right]+\mathcal{S}_{9}-\mathcal{S}_{7}\right)$ \\
& & $s_{9}$ & $\mathcal{O}\left(\mathcal{S}_{9}\right)$
\end{tabular}

Here, in the first column, we have also summarized the line bundles of the homogeneous coordinates on $d P_{2}$. 
The rational sections $\hat{s}_{P}, \hat{s}_{Q}$ and $\hat{s}_{R}$ are given by $e_{2}=0, e_{1}=0$ and $u=0$ in $\hat{X}$. From this it follows that their classes, denoted by capital letters, are given by:

$$
S_{P}=E_{2}, \quad S_{Q}=E_{1}, \quad S_{R}=H-E_{1}-E_{2}+\mathcal{S}_{9}+\left[K_{B}\right]
$$

as in [1]. We choose $\hat{s}_{P}$ as the zero section of the elliptic fibration of $\hat{X}$.

From the assignments 2.6 we see that the divisors $\mathcal{S}_{7}, \mathcal{S}_{9}$ are precisely the vanishing loci of the sections $s_{7}, s_{9}$ in (2.2), which is the reason for the choice of their labels. As mentioned before, these two divisors geometrically encode the intersections of the rational sections. Indeed, we observe the following intersections:

$$
\mathcal{S}_{7}=\pi\left(S_{Q} \cdot S_{R}\right), \quad \mathcal{S}_{9}=\pi\left(S_{P} \cdot S_{R}\right), \quad 0=\pi\left(S_{P} \cdot S_{Q}\right),
$$

where $\pi$ denotes the projection to the (co-)homology of the base $B$.

The assignment of sections in [1] for the base $B=\mathbb{P}^{2}$ can be recovered from (2.6) by making the replacements $\left[K_{B}^{-1}\right] \rightarrow 3 H_{B}, \mathcal{S}_{9} \rightarrow n_{2} H_{B}$ and $\mathcal{S}_{7} \rightarrow n_{12} H_{B}$, where $H_{B}$ is the hyperplane class of the base.

\subsection{Hypermultiplet Matter Representations and Multiplicities}

As mentioned in the Introduction, locally the fiber degenerates in the same fashion at codimension two as for the case $B=\mathbb{P}^{2}$. Furthermore, the intersections of the rational sections with the components of the reducible fiber do not depend on the global geometry of $B$. This implies that the $U(1)$-charges of matter fields are independent of the base $B$, as can be seen from the charge formula

$$
q_{m}=\left(S_{m}-S_{P}\right) \cdot c_{\mathrm{mat}},
$$

where $c_{\text {mat }}$ is an isolated $\mathbb{P}^{1}$ in the fiber corresponding to a matter field and where we collectively labeled the divisor classes $S_{m}=\left(S_{Q}, S_{R}\right)_{m}$, with $m=1,2$.

Furthermore, the functional dependencies of the polynomials describing the codimension two loci supporting matter on the sections $s_{i}$ are base-independent. However, the multiplicities of matter do depend on $B$ because the line bundles in which the $s_{i}$ 's take values are base dependent, see (2.6). Nevertheless, in the following we can still calculate the matter multiplicities directly for an arbitrary base by applying the results in Section 4 of [1] and the intersection theory on $B$.

First, we calculate the multiplicities of the singlets in Section 4.3 of [1]. These arise at codimension two singularities of the fibration due to the rational sections being illdefined. These codimension two loci are complete intersections in the base $B$ and their multiplicities are the number of intersection points given by

\begin{tabular}{|c|c|c|}
\hline Loci & Representation & Multiplicity \\
\hline$s_{7}=s_{3}=0$ & $\mathbf{1}_{(-1,1)}$ & $\mathcal{S}_{7} \cdot\left(\left[K_{B}^{-1}\right]+\mathcal{S}_{7}-\mathcal{S}_{9}\right)$ \\
\hline$s_{7}=s_{9}=0$ & $\mathbf{1}_{(0,2)}$ & $\mathcal{S}_{7} \cdot \mathcal{S}_{9}$ \\
\hline$s_{9}=s_{8}=0$ & $\mathbf{1}_{(-1,-2)}$ & $\mathcal{S}_{9} \cdot\left(\left[K_{B}^{-1}\right]+\mathcal{S}_{9}-\mathcal{S}_{7}\right)$ \\
\hline
\end{tabular}


where we used (2.6). Here - denotes the intersection product on $B$.

Second, we calculate the multiplicities of the singlets in Sections 4.1 and 4.2 of [1]. These arise at codimension two singularities where a rational section collides with a singularity in the Weierstrass fibration. We first calculate the line bundles of the polynomials of the complete intersections that contain the respective codimension two loci. They read

\begin{tabular}{|c|c||c|c|}
\hline Polynomial & Bundle & Polynomial & Bundle \\
\hline$\delta g_{6}^{\prime}$ & $\mathcal{O}\left(2\left[K_{B}^{-1}\right]+2 \mathcal{S}_{9}-\mathcal{S}_{7}\right)$ & $g_{9}^{\prime}$ & $\mathcal{O}\left(3\left[K_{B}^{-1}\right]+\mathcal{S}_{9}-\mathcal{S}_{7}\right)$ \\
\hline$g_{9}^{Q}$ & $\mathcal{O}\left(3\left[K_{B}^{-1}\right]\right)$ & $\hat{g}_{12}^{Q}$ & $\mathcal{O}\left(4\left[K_{B}^{-1}\right]\right)$ \\
\hline$g_{9+3 n_{2}}^{R}$ & $\mathcal{O}\left(3\left[K_{B}^{-1}\right]+3 \mathcal{S}_{9}\right)$ & $\hat{g}_{12+4 n_{2}}^{R}$ & $\mathcal{O}\left(4\left[K_{B}^{-1}\right]+4 \mathcal{S}_{9}\right)$ \\
\hline
\end{tabular}

The polynomials $\delta g_{6}^{\prime}$ and $g_{9}^{\prime}$ were defined in eq. (4.19) of [1]. Their zero loci contain the loci of the representation $\mathbf{1}_{(1,1)}$. Similarly, $g_{9}^{Q}$ and $\hat{g}_{12}^{Q}$ were defined in eqs. (4.6) and (4.7) of [1], respectively. Their zero loci contain the loci of the representation $\mathbf{1}_{(1,0)}$. Finally, the loci of $\mathbf{1}_{(0,1)}$ are contained in the zero loci of $g_{9+3 n_{2}}^{R}$ and $\hat{g}_{12+4 n_{2}}^{R}$ defined in eqs. (4.10) and (4.11) of [1], respectively.

The polynomials 2.11 also have zeros at the loci corresponding to the fields in 2.10 that have already been counted. We have to subtract these zeros with the appropriate order in order to obtain the multiplicities of the matter in Sections 4.1 and 4.2 of [1]. Because the functional dependencies of the polynomials in (2.11) on the $s_{i}$ 's are the same as for the case $B=\mathbb{P}^{2}$, the order of the zeroes determined in Section 4.4 of [1], using resultant techniques, still holds. Invoking these results, the rest of the multiplicities are

\begin{tabular}{|c|c|}
\hline Representation & Multiplicity \\
\hline $\mathbf{1}_{(1,0)}$ & $6\left[K_{B}^{-1}\right]^{2}+\left[K_{B}^{-1}\right] \cdot\left(4 \mathcal{S}_{7}-5 \mathcal{S}_{9}\right)-2 \mathcal{S}_{7}^{2}+\mathcal{S}_{7} \cdot \mathcal{S}_{9}+\mathcal{S}_{9}^{2}$ \\
\hline $\mathbf{1}_{(0,1)}$ & $6\left[K_{B}^{-1}\right]^{2}+\left[K_{B}^{-1}\right] \cdot\left(4 \mathcal{S}_{7}+4 \mathcal{S}_{9}\right)-2 \mathcal{S}_{7}^{2}-2 \mathcal{S}_{9}^{2}$ \\
\hline $\mathbf{1}_{(1,1)}$ & $6\left[K_{B}^{-1}\right]^{2}+\left[K_{B}^{-1}\right] \cdot\left(-5 \mathcal{S}_{7}+4 \mathcal{S}_{9}\right)+\mathcal{S}_{7}^{2}+\mathcal{S}_{7} \cdot \mathcal{S}_{9}-2 \mathcal{S}_{9}^{2}$ \\
\hline
\end{tabular}

We note that the results in Section 4 of [1] for the special base $B=\mathbb{P}^{2}$ are recovered by making the identifications $\left[K_{B}^{-1}\right] \rightarrow 3 H_{B}, \mathcal{S}_{7} \rightarrow n_{12} H_{B}, \mathcal{S}_{9} \rightarrow n_{2} H_{B}$ and $H_{B}^{2} \rightarrow 1$, where again $H_{B}$ is the hyperplane class of the base $B$.

\subsection{Anomaly Cancellation}

With the hypermultiplet representations and their multiplicities at hand, six-dimensional anomaly cancellation can be verified. For the convenience of the reader we summarize below the expressions for the respective mixed Abelian-gravitational, pure Abelian and pure gravitational anomaly cancellation: 1

\footnotetext{
${ }^{1}$ For further details, see Section 5 of [1].
} 


$$
\begin{aligned}
K_{B} \cdot b_{m n} & =-\frac{1}{6} \sum_{\underline{q}} x_{q_{m}, q_{n}} q_{m} q_{n}, \\
b_{m n} \cdot b_{k l}+b_{m k} \cdot b_{n l}+b_{m l} \cdot b_{n k}= & \sum_{\underline{q}} x_{q_{m}, q_{n}, q_{k}, q_{l}} q_{m} q_{n} q_{k} q_{l}, \\
273=H-V+29 T, & K_{B} \cdot K_{B}=9-T .
\end{aligned}
$$

Here $x_{q_{m}, q_{n}}$ and $x_{q_{m}, q_{n}, q_{k}, q_{l}}$ denote the number of matter hypermultiplets with charges $\left(q_{m}, q_{n}\right)$ and $\left(q_{m}, q_{n}, q_{k}, q_{l}\right)$ under $\mathrm{U}(1)_{m} \times \mathrm{U}(1)_{n}$, respectively, $\mathrm{U}(1)_{m} \times \mathrm{U}(1)_{n} \times \mathrm{U}(1)_{k} \times$ $\mathrm{U}(1)_{l}$. In addition, $H, V$ and $T$ denote the total number of hyper-, vector- and tensormultiplets, respectively. The $b_{m n}$ denote curves in the base $B$ defined as

$$
b_{m n}=-\pi\left(\sigma\left(\hat{s}_{m}\right) \cdot \sigma\left(\hat{s}_{n}\right)\right)=\left(\begin{array}{cc}
-2\left[K_{B}\right] & \mathcal{S}_{9}-\mathcal{S}_{7}-\left[K_{B}\right] \\
\mathcal{S}_{9}-\mathcal{S}_{7}-\left[K_{B}\right] & 2\left(\mathcal{S}_{9}-\left[K_{B}\right]\right)
\end{array}\right)_{m n} .
$$

Here we have collectively denoted rational sections $\hat{s}_{m}=\left(\hat{s}_{Q}, \hat{s}_{R}\right)_{m}$ and their divisor classes $S_{m}=\left(S_{Q}, S_{R}\right)_{m}$, with $m=1,2$ as before. The Shioda map of rational sections $\hat{s}_{m}$ to corresponding divisor classes $\sigma\left(\hat{s}_{m}\right)$ gives:

$$
\sigma\left(\hat{s}_{Q}\right)=S_{Q}-S_{P}-\left[K_{B}^{-1}\right], \quad \sigma\left(\hat{s}_{R}\right)=S_{R}-S_{P}-\left[K_{B}^{-1}\right]-\mathcal{S}_{9}
$$

See Section 2 of [1] as well as Section 2 of [2] for further details. In (2.14) we have further used (2.8).

Inserting 2.14 as well as the multiplicities 2.10 and 2.12 into the anomaly cancellation equations (2.13), we see that all equations are satisfied and thus all anomalies are cancelled. This verifies the six-dimensional anomaly cancellation for the general F-theory compactification with $\mathrm{U}(1) \times \mathrm{U}(1)$ gauge symmetry over an arbitrary base $B:^{2}$

\section{Compactifications for a General Base: $\mathrm{SU}(5) \times \mathrm{U}(1)^{2}$}

In Section 6.3 of [1] an example with $\mathrm{SU}(5) \times \mathrm{U}(1) \times \mathrm{U}(1)$ gauge symmetry was constructed as a non-generic elliptic Calabi-Yau threefold with $B=\mathbb{P}^{2}$. It was described as a toric hypersurface in a four-dimensional toric ambient space obtained by a toric resolution corresponding to one particular SU(5)-Top. In this Section we generalize this construction to an elliptically fibered Calabi-Yau threefold with an arbitrary base $B$.

In order to align the notation with the one of [2], we interchange the variables $d_{2}$ and $d_{4}$ in [1] and their respective divisor classes. After this change, the nodes of the affine Dynkin diagram of SU(5) correspond to $d_{0}, \ldots, d_{4}$ in cyclic order starting with the extended node, see the left figure in Figure 1.

\footnotetext{
${ }^{2}$ The base $B$ has to admit a generic elliptic fibration of the form (2.1), i.e. all line bundles in (2.6) have to have generic sections $s_{i}$. See the discussion in Section 2 of [2].
} 
For a specific choice [1] of a non-generic elliptic fibrations, where the following sections $s_{i}$ are chosen to vanish at $z_{2}=0$ in the following way:

$$
s_{1}=z_{2}^{3} s_{1}^{\prime}, \quad s_{2}=z_{2}^{2} s_{2}^{\prime}, \quad s_{3}=z_{2}^{2} s_{3}^{\prime}, \quad s_{5}=z_{2} s_{5}^{\prime},
$$

there is an $A_{4}$ singularity on top of the hyperplane divisor $\mathcal{S}_{\mathrm{SU}(5)}^{b}=H_{B}$ in $B=\mathbb{P}^{2}$, thus resulting in the $\mathrm{SU}(5)$ gauge symmetry at $z_{2}=0$. This was confirmed by computing the discriminant $\Delta$ of the Weierstrass fibration, which at $z_{2}=0$ takes the form

$$
\Delta=-z_{2}^{5}\left(\beta_{5}^{4} P+z_{2} \beta_{5}^{2} P_{2} R+\mathcal{O}\left(z_{2}^{2}\right)\right)
$$

where

$$
\beta_{5}=s_{6}, \quad P:=P_{1} P_{2} P_{3} P_{4} P_{5}=\left(s_{2}^{\prime} s_{5}^{\prime}-s_{1}^{\prime} s_{6}\right) s_{7}\left(s_{2}^{\prime} s_{7}-s_{3}^{\prime} s_{6}\right) s_{8}\left(s_{6} s_{9}-s_{7} s_{8}\right),
$$

and $R$ is a polynomial in $s_{i}$ 's with no common factors. We refer to the discussion around eq. (6.17) of [1] for further details.

The $A_{4}$ singularity is resolved by four blow-ups in the four- dimensional ambient space (2.2) with $B=\mathbb{P}^{2}$ :

$$
\pi_{\mathrm{SU}(5)}: \quad w=d_{1} d_{2}^{2} d_{3}^{3} d_{4}^{2} \tilde{w}, \quad v=d_{1} d_{2} d_{3} d_{4} \tilde{v}, \quad u=d_{3} d_{4} \tilde{u}, \quad z_{2}=d_{1} d_{2} d_{3} d_{4} \tilde{z} .
$$

Here we introduced the sections $d_{i}$ of the divisor bundles $\mathcal{O}\left(D_{i}\right)$ associated with the exceptional divisors $D_{i}:=\left\{d_{i}=0\right\}$ of the resolution. The Stanley-Reissner ideal of this resolution reads

$$
\begin{aligned}
S R= & \pi_{\mathrm{SU}(5)}^{*}\left(S R_{B}\right) \cup\left\{v e_{1}, e_{1} e_{2}, u v, u w, w e_{2}\right\} \cup\left\{z_{2} d_{3}, d_{1} d_{3}, d_{1} d_{4}\right\} \cup\left\{d_{1} e_{1}, z_{2} e_{1},\right. \\
& \left.d_{2} e_{1}, d_{4} e_{1}, d_{1} u, d_{2} u, d_{1} e_{2}, d_{2} e_{2}, d_{3} e_{2}, d_{4} e_{2}, z_{2} w, d_{1} w, d_{4} w, d_{3} v, d_{4} v, z_{2} d_{2} v\right\},
\end{aligned}
$$

where we pulled back the Stanley-Reissner ideal $S R_{B}$ of the base using (3.4).

Along the same lines as in the previous Section it is possible to generalize this example to elliptic Calabi-Yau threefolds $\hat{X}_{\mathrm{SU}(5)}$ over an arbitrary base. The threefold $\hat{X}_{\mathrm{SU}(5)}$ is still described as a hypersurface in the blow-up of $(2.2)$ via $(3.4)$ for a general $B$. We denote this new ambient space as $\widehat{d P}_{2}^{B}\left(\mathcal{S}_{7}, \mathcal{S}_{9}\right)$. The divisor $\mathcal{S}_{\mathrm{SU}(5)}^{b}$, which supports the $A_{4}$ singularity, is replaced by a general divisor in the base $B$ associated with a section $z$, replacing the section $z_{2}$ above.

In the elliptic Calabi-Yau threefold $\hat{X}_{\mathrm{SU}(5)}$ over a general base $B$, the sections $s_{i}^{\prime}$ $(i=1,2,3,5)$ are promoted to sections of the following line bundles:

\begin{tabular}{c|c} 
Section & Bundle \\
\hline$s_{1}^{\prime}$ & $\mathcal{O}\left(3\left[K_{B}^{-1}\right]-\mathcal{S}_{7}-\mathcal{S}_{9}-3 \mathcal{S}_{\mathrm{SU}(5)}\right)$ \\
$s_{2}^{\prime}$ & $\mathcal{O}\left(2\left[K_{B}^{-1}\right]-\mathcal{S}_{9}-2 \mathcal{S}_{\mathrm{SU}(5)}\right)$ \\
$s_{3}^{\prime}$ & $\mathcal{O}\left(\left[K_{B}^{-1}\right]+\mathcal{S}_{7}-\mathcal{S}_{9}-2 \mathcal{S}_{\mathrm{SU}(5)}\right)$ \\
$s_{5}^{\prime}$ & $\mathcal{O}\left(2\left[K_{B}^{-1}\right]-\mathcal{S}_{7}-\mathcal{S}_{\mathrm{SU}(5)}\right)$
\end{tabular}


by the Calabi-Yau condition. Here we introduced the vertical divisor $\mathcal{S}_{\mathrm{SU}(5)}=\pi^{*}\left(\mathcal{S}_{\mathrm{SU}(5)}^{b}\right)$

in $\hat{X}_{\mathrm{SU}(5)}$. This can be seen by first performing the proper transform of (2.1) under (3.4) using (3.1) and then computing the anti-canonical bundle of the blown-up ambient space (2.2), which reads

$$
K_{\widehat{d P}_{2}^{B}}^{-1}=\pi_{\mathrm{SU}(5)}^{*}\left(K_{d P_{2}^{B}}^{-1}\right)-2 D_{1}-3 D_{2}-5 D_{3}-4 D_{4}
$$

Here $\pi_{\mathrm{SU}(5)}^{*}$ is the pullback (3.4) and we refer also to Appendix C of [2] for more details on the total Chern class of $\widehat{d P}_{2}^{B}\left(\mathcal{S}_{7}, \mathcal{S}_{9}\right)$. Requiring $\hat{X}_{\mathrm{SU}(5)}$ to be a section of $K_{\widehat{d P}_{2}^{B}}^{-1}$ we immediately obtain the assignments (3.6).

\subsection{Hypermultiplet Matter Representations}

As pointed out in Sections 2 the hypermultiplet matter representations do not depend on the choice of the base, and for the non-generic elliptic fibration (3.1) the representations of $\mathrm{SU}(5) \times \mathrm{U}(1) \times \mathrm{U}(1)$ were subsequently determined in [2]. In this Subsection we therefore present only a summary of the key results and refer the reader to Section 6 of [2] for the details of these calculations.

We first summarize the intersection numbers on $\hat{X}_{\mathrm{SU}(5)}$, which can be calculated using the representation of the even-dimensional cohomology ring $H^{(*, *)}\left(\hat{X}_{\mathrm{SU}(5)}\right)$ as the quotient polynomial ring in the divisors $\left\{S_{P}, S_{Q}, S_{R}, D_{i}, D_{\alpha}\right\}$ modulo the Stanley-Reissner ideal (3.5) with $z_{2} \rightarrow z$. With the full cohomology ring at hand the intersection matrix of the exceptional divisors over an arbitrary point of $\mathcal{S}_{\mathrm{SU}(5)}$ is determined as

$$
D_{I} \cdot D_{J} \cdot D_{\alpha}=-C_{I J} \mathcal{S}_{\mathrm{SU}(5)} \cdot S_{P} \cdot D_{\alpha}, \quad\left(-C_{I J}\right)=\left(\begin{array}{ccccc}
-2 & 1 & 0 & 0 & 1 \\
1 & -2 & 1 & 0 & 0 \\
0 & 1 & -2 & 1 & 0 \\
0 & 0 & 1 & -2 & 1 \\
1 & 0 & 0 & 1 & -2
\end{array}\right)
$$

where $I$ and $J$ are in the range $\{0, \ldots, 4\}$ with $D_{0}$ as the extended node, and $D_{\alpha}$ is an arbitrary vertical divisor. The divisors $d_{i}=0, i=1, \ldots, 4$, that are $\mathbb{P}^{1}$-fibrations over $\mathcal{S}_{\mathrm{SU}(5)}^{b}$ are the Cartan divisors of $\mathrm{SU}(5)$. The individual $\mathbb{P}^{1}$ 's in the fiber are curves $c_{-\alpha_{i}}$ that correspond to the simple roots $\alpha_{i}$ of SU(5), with $\alpha_{0}=-\sum_{i} \alpha_{i}$ the extended node; the curves intersect as the affine Dynkin diagram of $\mathrm{SU}(5)$, thus justifying the depiction by the left figure of Figure 1. The Shioda map is of the following form:

$$
\sigma\left(\hat{s}_{Q}\right)=S_{Q}-S_{P}-\left[K_{B}^{-1}\right]+\frac{1}{5}\left(2 D_{1}+4 D_{2}+6 D_{3}+3 D_{4}\right), \quad \sigma\left(\hat{s}_{R}\right)=S_{R}-S_{P}-\left[K_{B}^{-1}\right]-\mathcal{S}_{9},
$$

which can also be shown using the presentation of $H^{(*, *)}\left(\hat{X}_{\mathrm{SU}(5)}\right)$ as a polynomial ring.

We now summarize the hypermultiplet matter representations located at codimension two singularities of the fibration. In addition to singlet representations of $\mathrm{SU}(5)$ summarized in the respective first columns in 2.10) and 2.12 there are also non-singlet 
representations. The latter ones appear at $z=P_{i}=0(i=1, \ldots, 5)$ and at $z=\beta_{5}=0$, where $P_{i}$ and $\beta_{5}$ are defined in $(3.3)$.

At $z=P_{i}=0(i=1, \ldots, 5)$ the singularity enhances to an $A_{5}$ singularity with local SU(6) symmetry, where one of the $\mathbb{P}^{1}$ 's at codimension one splits into a sum of two isolated $\mathbb{P}^{1}$ 's. By calculating the $\mathrm{SU}(5)$-weight of the split curve, the matter located at these loci can be seen to transform as the 5 representation of $\mathrm{SU}(5)$. As an example, see the center figure of Figure 1 where the Dynkin diagram of the fiber at $z=P_{1}=0$ is shown. The $\mathrm{U}(1)$-charges of the $\mathbf{5}$ representations at each $P_{i}=0$ are calculated as the intersections of the isolated rational curves, corresponding to the weight one of the $\mathbf{5}$ representation, and the Shioda map (3.9). The results are summarized in the following Table:

\begin{tabular}{|c||c|c|c|c|c|}
\hline Loci & $z=P_{1}=0$ & $z=P_{2}=0$ & $z=P_{3}=0$ & $z=P_{4}=0$ & $z=P_{5}=0$ \\
\hline$\left(q_{1}, q_{2}\right)$ & $\left(-\frac{2}{5}, 0\right)$ & $\left(-\frac{2}{5}, 1\right)$ & $\left(\frac{3}{5}, 0\right)$ & $\left(\frac{3}{5}, 1\right)$ & $\left(-\frac{2}{5},-1\right)$ \\
\hline
\end{tabular}

At the single locus $z=\beta_{5}=0$, the singularity enhances to a $D_{5}$ singularity with local enhancement to $\mathrm{SO}(10)$ symmetry. The representation at this locus is $\mathbf{1 0}_{(1 / 5,0)}$. The splitting of the rational curves and their intersections over this codimension two locus is depicted on the right figure of Figure 1 .
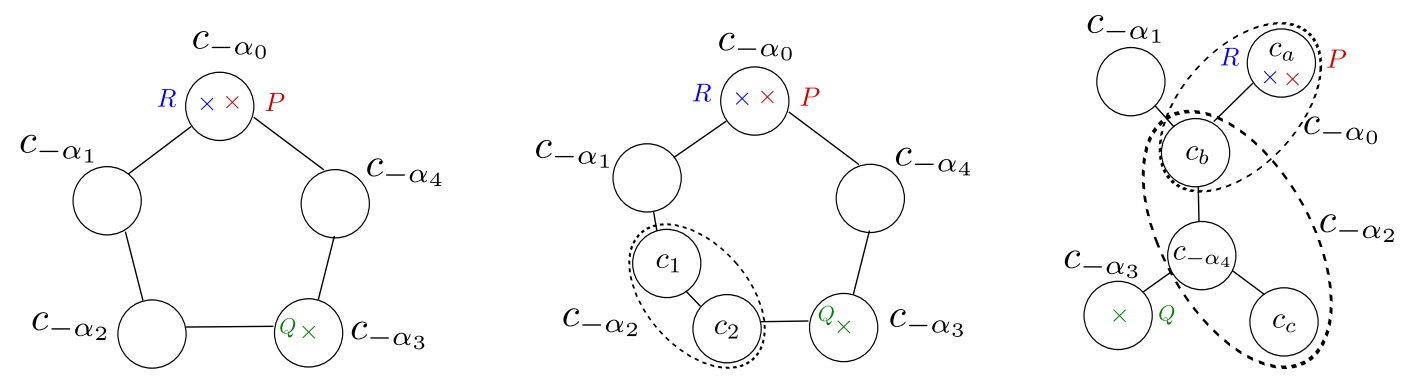

Figure 1: Left: $I_{4}$ singularity over a generic point of the divisor $z=0$, marked according to the interaction of the nodes with $\left\{S_{P}, S_{Q}, S_{R}\right\}$. Center: $A_{5}$ singularity with local SU(6) enhancement at $z=P_{1}=0$. Right: $D_{5}$ singularity with local $\mathrm{SO}(10)$ enhancement at $z=\beta_{5}=0$. The dashed lines indicate nodes of the Dynkin diagram of $\mathrm{SU}(5)$ that split at a codimension two locus under consideration.

\subsection{Hypermultiplet Matter Multiplicities}

In this Subsection we determine the multiplicities of all hypermultiplet representations for an arbitrary base $B$.

We begin with the multiplicities of the $\mathrm{SU}(5)$-singlets 2.10 . We note that there is only a minor change due to the presence of the $\mathrm{SU}(5)$ gauge symmetry since the line 
bundle of $s_{3}^{\prime}$ is now given by (3.6). Thus, we immediately obtain:

\begin{tabular}{|c|c|c|}
\hline Loci & Representation & Multiplicity \\
\hline$s_{7}=s_{3}^{\prime}=0$ & $\mathbf{1}_{(-1,1)}$ & $\mathcal{S}_{7} \cdot\left(\left[K_{B}^{-1}\right]+\mathcal{S}_{7}-\mathcal{S}_{9}-2 \mathcal{S}_{\mathrm{SU}(5)}\right)$ \\
\hline$s_{7}=s_{9}=0$ & $\mathbf{1}_{(0,2)}$ & $\mathcal{S}_{7} \cdot \mathcal{S}_{9}$ \\
\hline$s_{9}=s_{8}=0$ & $\mathbf{1}_{(-1,-2)}$ & $\mathcal{S}_{9} \cdot\left(\left[K_{B}^{-1}\right]+\mathcal{S}_{9}-\mathcal{S}_{7}\right)$ \\
\hline
\end{tabular}

The multiplicities of the $\mathbf{5}$ representations and the $\mathbf{1 0}$ representation are calculated straightforwardly by counting the points in the loci $z=P_{i}=0(i=1, \cdots, 5)$ and $z=\beta_{5}=0$, respectively. Since these loci are complete intersections, the results read:

\begin{tabular}{|c|c|c|}
\hline Loci & Representation & Multiplicity \\
\hline$z=P_{1}=0$ & $\boldsymbol{5}_{(-2 / 5,0)}$ & $\mathcal{S}_{\mathrm{SU}(5)} \cdot\left(4\left[K_{B}^{-1}\right]-\mathcal{S}_{7}-\mathcal{S}_{9}-3 \mathcal{S}_{\mathrm{SU}(5)}\right)$ \\
\hline$z=P_{2}=0$ & $\mathbf{5}_{(-2 / 5,1)}$ & $\mathcal{S}_{\mathrm{SU}(5)} \cdot \mathcal{S}_{7}$ \\
\hline$z=P_{3}=0$ & $\mathbf{5}_{(3 / 5,0)}$ & $\mathcal{S}_{\mathrm{SU}(5)} \cdot\left(2\left[K_{B}^{-1}\right]-\mathcal{S}_{9}+\mathcal{S}_{7}-2 \mathcal{S}_{\mathrm{SU}(5)}\right)$ \\
\hline$z=P_{4}=0$ & $\mathbf{5}_{(3 / 5,1)}$ & $\mathcal{S}_{\mathrm{SU}(5)} \cdot\left(\left[K_{B}^{-1}\right]+\mathcal{S}_{9}-\mathcal{S}_{7}\right)$ \\
\hline$z=P_{5}=0$ & $\mathbf{5}_{(-2 / 5,-1)}$ & $\mathcal{S}_{\mathrm{SU}(5)} \cdot\left(\left[K_{B}^{-1}\right]+\mathcal{S}_{9}\right)$ \\
\hline$z=\beta_{5}=0$ & $\mathbf{1 0}_{(1 / 5,0)}$ & $\mathcal{S}_{\mathrm{SU}(5)} \cdot\left[K_{B}^{-1}\right]$ \\
\hline
\end{tabular}

Here we have made use of (2.6) and (3.6).

The multiplicities of the remaining singlets 2.12 have to be calculated with special care. Similarly, as in the pure $\mathrm{U}(1) \times \mathrm{U}(1)$ case, some of the non-Abelian representations are contained in the loci of the singlets, i.e. the loci of the non-Abelian matter are roots of the polynomials in 2.11. Thus, in order to avoid a double-counting, we have to subtract the fields we have already counted. For instance, the multiplicity of the fields in the representation $\mathbf{1}_{(1,1)}$ is obtained from the number of points in the complete intersection $\delta g_{6}^{\prime}=g_{9}^{\prime}=0$ after subtracting a fourth order zero, a third order zero and a double zero corresponding to the representation $\mathbf{1}_{(-1,-2)}, \mathbf{5}_{(3 / 5,1)}$ and $\mathbf{5}_{(-2 / 5,1)}$, respectively.

The determination of the multiplicity for the representation $\mathbf{1}_{(1,0)}$ is more involved because the polynomial equations $g_{9}^{Q}=\hat{g}_{12}^{Q}=0$, which contain this representation, vanish automatically along the GUT-divisor $\mathcal{S}_{\mathrm{SU}(5)}$ at $z=0$. Thus, in order to determine its multiplicity we have to cancel global factors of $z$ in both $g_{9}^{Q}=\hat{g}_{12}^{Q}=0$. Then we can count the number of points in this complete intersection, where we have to subtract zeroes of order one corresponding to the representations $\mathbf{1}_{(1,1)}, \mathbf{1}_{(-1,1)}, \mathbf{1}_{(-1,-2)}, \mathbf{5}_{(3 / 5,1)}$ and $\mathbf{5}_{(3 / 5,0)}$.

Finally, in order to obtain the multiplicity of the representation $\mathbf{1}_{(0,1)}$ we have to count the number of points in $g_{6}^{R}=g_{9}^{R}=0$ by subtracting the zeros of order one, sixteen, and five corresponding to the representations $\left\{\mathbf{1}_{(1,1)}, \mathbf{1}_{(-1,1)}\right\},\left\{\mathbf{1}_{(0,2)}, \mathbf{1}_{(-1,-2)}\right\}$, and $\left\{\boldsymbol{5}_{(-2 / 5,1)}, \mathbf{5}_{(3 / 5,-1)}, \mathbf{5}_{(-2 / 5,-1)}\right\}$, respectively. 
We summarize the results of this analysis in the following Table:

\begin{tabular}{|c|c|}
\hline Representation & Multiplicity \\
\hline $\mathbf{1}_{(1,0)}$ & $6\left[K_{B}^{-1}\right]^{2}+\left[K_{B}^{-1}\right] \cdot\left(-12 \mathcal{S}_{\mathrm{SU}(5)}+4 \mathcal{S}_{7}-5 \mathcal{S}_{9}\right)$ \\
& $+6 \mathcal{S}_{\mathrm{SU}(5)}^{2}+\mathcal{S}_{\mathrm{SU}(5)} \cdot\left(-\mathcal{S}_{7}+5 \mathcal{S}_{9}\right)-2 \mathcal{S}_{7}^{2}+\mathcal{S}_{7} \cdot \mathcal{S}_{9}+\mathcal{S}_{9}^{2}$ \\
\hline $\mathbf{1}_{(0,1)}$ & $6\left[K_{B}^{-1}\right]^{2}+\left[K_{B}^{-1}\right] \cdot\left(-5 \mathcal{S}_{\mathrm{SU}(5)}+4 \mathcal{S}_{7}+4 \mathcal{S}_{9}\right)$ \\
& $-\mathcal{S}_{\mathrm{SU}(5)} \cdot\left(\mathcal{S}_{7}+5 \mathcal{S}_{9}\right)-2 \mathcal{S}_{7}^{2}-2 \mathcal{S}_{9}^{2}$ \\
\hline $\mathbf{1}_{(1,1)}$ & $\begin{array}{c}6\left[K_{B}^{-1}\right]^{2}+\left[K_{B}^{-1}\right] \cdot\left(-5 \mathcal{S}_{\mathrm{SU}(5)}-5 \mathcal{S}_{7}+4 \mathcal{S}_{9}\right) \\
\end{array}$ \\
& $+\mathcal{S}_{\mathrm{SU}(5)} \cdot\left(3 \mathcal{S}_{7}-5 \mathcal{S}_{9}\right)+\mathcal{S}_{7}^{2}+\mathcal{S}_{7} \cdot \mathcal{S}_{9}-2 \mathcal{S}_{9}^{2}$ \\
\hline
\end{tabular}

Finally, we note the presence of additional hypermultiplets for a non-simply connected GUT-divisor $\mathcal{S}_{\mathrm{SU}(5)}^{b}$. The divisor $\mathcal{S}_{\mathrm{SU}(5)}^{b}$ is in general a curve of genus $g$ and adds $x_{A d j}=g$ matter hypermultiplets in the adjoint representation 25 of SU(5) [7], which are uncharged under the $\mathrm{U}(1)$ gauge fields:

\begin{tabular}{|c|c|}
\hline Representation & Multiplicity \\
\hline $\mathbf{2 4}_{(0,0)}$ & $g$ \\
\hline
\end{tabular}

We note that given an arbitrary divisor $\mathcal{S}_{\mathrm{SU}(5)}^{b}$ the genus $g$ is calculated via adjunction as

$$
K_{B} \cdot \mathcal{S}_{\mathrm{SU}(5)}^{b}+\left(\mathcal{S}_{\mathrm{SU}(5)}^{b}\right)^{2}=2 g-2
$$

\subsection{Anomaly cancellation}

The Abelian anomaly cancellation equations in (2.13) can readily be evaluated by including the non-Abelian representations with their respective multiplicities and their number of weights. However the curves $b_{m n}$ in the base are now modified by the presence of the $\mathcal{S}_{\mathrm{SU}(5)}$ divisor, and are given by

$$
b_{m n}=-\pi\left(\sigma\left(\hat{s}_{n}\right) \cdot \sigma\left(\hat{s}_{m}\right)\right)=b_{m n}^{0}+\left(C^{-1}\right)^{i j}\left(S_{m} \cdot c_{-\alpha_{i}}\right)\left(S_{n} \cdot c_{-\alpha_{j}}\right) \mathcal{S}_{\mathrm{SU}(5)},
$$

where the Shioda map $\sigma\left(\hat{s}_{m}\right)(m=1,2)$ is given in $(3.9), b_{m n}^{0}$ refers to the curves in (2.14), i.e. without $\mathcal{S}_{\mathrm{SU}(5)},\left(C^{-1}\right)^{i j}$ is the inverse Cartan matrix of $\mathrm{SU}(5)$ (see (3.8)) and $c_{-\alpha_{i}}$ are the fibral curves corresponding to the simple roots of $\mathrm{SU}(5)$. After further manipulations, 3.16 can be cast in the following form:

$$
b_{m n}=\left(\begin{array}{cc}
-2\left[K_{B}\right]-\frac{6}{5} \mathcal{S}_{\mathrm{SU}(5)} & \mathcal{S}_{9}-\mathcal{S}_{7}-\left[K_{B}\right] \\
\mathcal{S}_{9}-\mathcal{S}_{7}-\left[K_{B}\right] & 2\left(\mathcal{S}_{9}-\left[K_{B}\right]\right)
\end{array}\right)_{m n} .
$$

Comparing the two sides in (2.13) we see that indeed all Abelian and mixed Abeliangravitational anomalies are cancelled. However, in the presence of a non-Abelian gauge 
sector there are additional anomaly equations, see for example [8]. They can be summarize as:

Non-Abelian-gravitational anomaly $F_{\kappa}^{2} R^{2}: \quad \frac{1}{6}\left(A_{A d j_{\kappa}}-\sum_{\mathbf{R}} x_{\mathbf{R}} A_{\mathbf{R}}\right)=K_{B}^{-1} \cdot\left(\frac{b_{\kappa}}{\lambda_{\kappa}}\right)$, Pure non-Abelian anomaly $F_{\kappa}^{4}: \quad B_{A d j_{\kappa}}-\sum_{\mathbf{R}} x_{\mathbf{R}} B_{\mathbf{R}}=0$, Pure non-Abelian anomaly $F_{\kappa}^{2} F_{\kappa}^{2}: \quad \frac{1}{3}\left(\sum_{\mathbf{R}} x_{\mathbf{R}} C_{\mathbf{R}}-C_{A d j_{\kappa}}\right)=\left(\frac{b_{\kappa}}{\lambda_{\kappa}}\right)^{2}$, Non-Abelian-Abelian anomaly $F_{\kappa}^{3} F_{U(1)_{i}}: \quad \sum_{\mathbf{R}, q_{i}} x_{\mathbf{R}, q_{i}} q_{i} E_{\mathbf{R}}=0$,

Non-Abelian-Abelian anomaly $F_{\kappa}^{2} F_{U(1) i} F_{U(1)_{j}}: \quad \sum_{\mathbf{R}, q_{i}, q_{j}} x_{\mathbf{R}, q_{i}, q_{j}} q_{i} q_{j} A_{\mathbf{R}}=\left(\frac{b_{\kappa}}{\lambda_{\kappa}}\right) \cdot b_{i j}$.

Here, the ' $x$.' variables denote the multiplicities of hypermultiplets: $x_{\mathbf{R}}, x_{\mathbf{R}, q_{i}}$ and $x_{\mathbf{R}, q_{i}, q_{j}}$ are the number of hypermultiplets in the $\mathbf{R}$ representation, in the $\mathbf{R}$ representation with charge $q_{i}$ under $\mathrm{U}(1)_{i}$ and in the $\mathbf{R}$ representation with charges $\left\{q_{i}, q_{j}\right\}$ under $\mathrm{U}(1)_{i} \times \mathrm{U}(1)_{j}$, respectively. Furthermore, we used the following group theory relations:

$$
\operatorname{tr}_{\mathbf{R}} F_{\kappa}^{2}=A_{\mathbf{R}} \operatorname{tr} F_{\kappa}^{2}, \quad \operatorname{tr}_{\mathbf{R}} F_{\kappa}^{3}=E_{\mathbf{R}} \operatorname{tr} F_{\kappa}^{3}, \quad \operatorname{tr}_{\mathbf{R}} F_{\kappa}^{4}=B_{\mathbf{R}} \operatorname{tr} F_{\kappa}^{4}+C_{\mathbf{R}}\left(\operatorname{tr} F_{\kappa}^{2}\right)^{2} .
$$

Here 'tr' denotes the trace with respect to the fundamental representation and $\operatorname{tr}_{\mathbf{R}}$ is the trace for a given representation $\mathbf{R}$. For $\kappa=\mathrm{SU}(N)$, the group theory factors (3.19) have the following values: $\mathrm{S}^{3}$

\begin{tabular}{|c|c|c|c|c|c|}
\hline Representation & Dimension & $A_{\mathbf{R}}$ & $B_{\mathbf{R}}$ & $C_{\mathbf{R}}$ & $E_{\mathbf{R}}$ \\
\hline Fundamental & $N$ & 1 & 1 & 0 & 1 \\
\hline Adjoint & $N^{2}-1$ & $2 N$ & $2 N$ & 6 & $2 N$ \\
\hline Antisymmetric & $N(N-1) / 2$ & $N-2$ & $N-8$ & 3 & 1 \\
\hline
\end{tabular}

Furthermore, $\lambda_{\kappa}$ in 3.18$)$ is the group normalization constant defined by $\lambda_{\kappa}=2 c_{G(I)} / E_{a d j_{\kappa}}$, where $c_{G(I)}$ is the dual Coxeter number and $E_{\mathbf{R}}$ is defined in $(3.19)$. For $\kappa=\mathrm{SU}(N)$, $\lambda_{\kappa}=1$. Finally, $b_{\kappa}$ in $(3.18)$ is the divisor supporting the non-Abelian group in the base; in our case $\kappa=\mathrm{SU}(5)$ and $b_{\kappa}=\mathcal{S}_{\mathrm{SU}(5)}$.

With the above information we can evaluate explicitly the anomaly cancellation equations (3.18). In our case we have to sum over the fundamental, anti-symmetric and adjoint representations, along with their multiplicities $(3.12)$ and (3.14), and the expression (3.17) for $b_{m n}$. The upshot is that all the anomaly cancellation equations (3.18) are satisfied. Thus, the generic F-theory compactification with the chosen $\mathrm{SU}(5) \times \mathrm{U}(1) \times \mathrm{U}(1)$ gauge symmetry over an arbitrary base $B$ is anomaly free.

\footnotetext{
${ }^{3}$ See [9] for further details.
} 


\section{Acknowledgments}

We would like to thank Thomas Grimm, Jim Halverson, Albrecht Klemm, Sakura SchäferNameki, Peng Song and Washington Taylor for discussions. We are especially indebted to Antonella Grassi for many useful discussions and comments. M.C. is grateful to the Theory Division of CERN for hospitality. D.K. thanks the University of Hamburg and DESY for hospitality. H.P is grateful to PiTP at the Institute for Advanced Study for hospitality. This research is supported in part by the DOE grant de-sc0007901 (M.C., H.P., D.K.), the NSF String Vacuum Project Grant No. NSF PHY05-51164 (H.P.), Dean's Funds for Faculty Working Group (M.C. and D.K.), the Fay R. and Eugene L. Langberg Endowed Chair (M.C.) and the Slovenian Research Agency (ARRS) (M.C.).

\section{References}

[1] M. Cvetič, D. Klevers, and H. Piragua, "F-Theory Compactifications with Multiple U(1)-Factors: Constructing Elliptic Fibrations with Rational Sections," arXiv: 1303.6970 [hep-th].

[2] M. Cvetič, A. Grassi, D. Klevers, and H. Piragua, "Chiral Four-Dimensional F-Theory Compactifications With SU(5) and Multiple U(1)-Factors," arXiv:1306.3987 [hep-th].

[3] J. Borchmann, C. Mayrhofer, E. Palti, and T. Weigand, "Elliptic fibrations for $\mathrm{SU}(5) \times \mathrm{U}(1) \times \mathrm{U}(1)$ F-theory vacua," arXiv:1303.5054 [hep-th].

[4] T. W. Grimm, A. Kapfer, and J. Keitel, "Effective action of 6D F-Theory with U(1) factors: Rational sections make Chern-Simons terms jump," arXiv:1305.1929 [hep-th].

[5] V. Braun, T. W. Grimm, and J. Keitel, "Geometric Engineering in Toric F-Theory and GUTs with U(1) Gauge Factors," arXiv:1306.0577 [hep-th].

[6] J. Borchmann, C. Mayrhofer, E. Palti, and T. Weigand, "SU(5) Tops with Multiple U(1)s in F-theory," arXiv:1307.2902 [hep-th].

[7] S. H. Katz, D. R. Morrison, and M. R. Plesser, "Enhanced gauge symmetry in type II string theory," Nucl.Phys. B477 (1996) 105-140, arXiv: hep-th/9601108 [hep-th].

[8] D. S. Park and W. Taylor, "Constraints on 6D Supergravity Theories with Abelian Gauge Symmetry," JHEP 1201 (2012) 141, arXiv:1110.5916 [hep-th].

[9] W. Taylor, "TASI Lectures on Supergravity and String Vacua in Various Dimensions," arXiv:1104.2051 [hep-th]. 